\title{
Urinary Tract Infection in Children: A Review of the Established Practice Guidelines
}

\author{
Authors: \\ *Samuel Uwaezuoke, Adaeze Ayuk, Uzoamaka Muoneke \\ Department of Paediatrics, University of Nigeria Teaching Hospital Ituku-Ozalla, \\ Enugu, Nigeria \\ *Correspondence to snuwaezuoke@yahoo.com \\ Disclosure: \\ The authors have declared no conflicts of interest. \\ Received: \\ 01.01.2020 \\ Accepted: \\ 20.02.2020 \\ Keywords: \\ Children, evaluation, guidelines, review, therapy, urinary tract infection (UTI). \\ Citation: \\ EMJ Microbiol Infect Dis. 2020;1[1]:57-65.
}

\section{Abstract}

Urinary tract infection (UTI) is a significant cause of morbidity in children. Delayed treatment is associated with complications that may result in chronic kidney disease and, subsequently, end-stage kidney disease. Over the years, clinical practice guidelines have advanced to ensure the best global practices in treating the infection and preventing its progression to chronic kidney disease. The established practice guidelines address five main questions: 1) which children should have their urine tested; 2) how the sample should be obtained; 3) which radiological tests are recommended after a diagnosis of UTI; 4) how the infection should be treated; 5) and how affected children should be followed up. There is a substantial overlap in the recommendations of the American Academy of Pediatrics (AAP) guidelines and the UK's National Institute for Health and Clinical Excellence (NICE) guidelines. Subtle differences, however, exist between the two established guidelines. An evidence-based paradigm shift of some traditional concepts about UTI in children has contributed to the revision and update of these guidelines. Further research is needed to clarify the role of host and genetic factors in renal scarring, as well as the diagnostic criteria for UTI. This narrative review aims to discuss the current recommendations of these established practice guidelines with an emphasis on the diagnosis, radiological investigation, treatment, and follow-up of UTI in children.

\section{INTRODUCTION}

Urinary tract infection (UTI) is a significant cause of morbidity in children.,2 Prevalence rates of a first-time symptomatic UTI are highest in both male and female infants during the first 12 months of life, with a marked reduction after this period. ${ }^{1}$ UTI is broadly categorised into upper UTI (pyelonephritis) and lower UTI (cystitis). The usual trajectory of UTI spread involves the ascension of periurethral organisms through the urethra into the bladder (cystitis). These

uropathogens then migrate upwards through the ureters to the renal parenchyma (pyelonephritis) and may be followed by haematogenous spread (bacteraemia). Pyelonephritis is associated with renal parenchymal scarring in approximately $10-30 \%$ of paediatric patients with febrile UTI. ${ }^{3}$ Renal scars can result in hypertension, chronic kidney disease (CKD), and subsequently endstage kidney disease. ${ }^{4}$ Over the years, clinical practice guidelines have advanced to ensure the best global practices in managing UTI and preventing progression to CKD. 
During childhood, $30-50 \%$ of children who have previously had an episode of UTI will have at least one recurrence. ${ }^{5}$ Predisposition to recurrence of UTI has recently been linked to genetic factors; the identified genes were HSPA 1B, CXCR1, CXCR2, TLR2, TLR4, and TGF- $\beta 1 .{ }^{6}$ However, nongenetic factors are also associated with infection risk and recurrence. These factors include constipation, severe acute malnutrition, obstructive uropathy, urolithiasis, absent circumcision in boys (particularly within the first year of life), and female sex after infancy. 7,8 Vesicoureteral reflux (VUR) also contributes to recurrence of UTI in children, which in turn promotes upper urinary tract involvement and renal scarring. Although postinfection renal scarring has traditionally been associated with subsequent $C K D$, its causal relationship with UTI-associated VUR has recently been challenged by some authors. ${ }^{9,10} \mathrm{~A}$ high incidence of scar formation in post-UTI patients without VUR was noted in one of the studies. ${ }^{9}$ The other report identified virulence of uropathogens, host defense factors, and genetic predisposition as risk factors for renal scarring. ${ }^{10}$ Many of the sequelae from scarring are now attributed to pre-existing intrinsic renal disease. " Specifically, VUR-related renal damage is now linked to congenital dysplastic kidneys ${ }^{12}$ and is regarded as part of the congenital anomaly of the kidney and urinary tract syndrome..$^{13}$

These findings have led to a shift in clinical practice guidelines which until now recommended routine voiding cystourethrography (VCUG) or micturating cystourethrography (MCUG) after a first febrile UTI episode. The 2011 American Academy of Pediatrics (AAP) guideline now recommends a VCUG (or MCUG) after the second episode of UTI, with deference to parent preferences. ${ }^{14}$ Similarly, the UK's National Institute of Health and Clinical Excellence (NICE) guideline dissuades clinicians from performing unnecessary invasive imaging tests and advocates a targeted investigative approach in high-risk children. ${ }^{15}$ However, because the prevention of renal scarring is the objective of all therapeutic approaches for childhood UTI, it has been suggested that children at high risk of post-UTI renal scarring should still be treated and investigated pending the accumulation of more evidence to support any paradigm shift. ${ }^{16}$ Clinical practice guidelines address five main questions: 1) which children should have their urine tested; 2) how the sample should be obtained; 3) which radiological tests are recommended after a diagnosis of UTI; 4) how the infection should be treated; 5) and how affected children should be followed up. This narrative review aims to discuss the current recommendations of the established practice guidelines with emphasis on the diagnosis, radiological investigation, treatment, and follow-up of UTI in children.

\section{DIAGNOSIS OF URINARY TRACT INFECTION}

Accurate diagnosis of UTI is critical for instituting appropriate treatment with antibiotics and preventing upper tract involvement and longterm renal disease. Proper interpretation of urinalysis and urine culture results form the basis for the diagnosis of UTI in children. Biomarkers of inflammation (such as pyuria and the presence of leukocyte esterase) and bacterial growth from urine cultures are usually required to establish a diagnosis. For the latter, which remains the gold standard, the reference standard for UTI diagnosis is a single uropathogen cultured from a specimen obtained at specific concentrations: $>10^{3}$ or 1,000 colony-forming units (CFU)/mL for a specimen from suprapubic aspiration (SPA); $>10^{4}$ or $10,000 \mathrm{CFU} / \mathrm{mL}$ for a catheter specimen; or $\geq 10^{5}$ or $10,000 \mathrm{CFU} / \mathrm{mL}$ for a 'clean-catch', midstream specimen. ${ }^{17}$ Although some authors advocate the use of lower colony counts in symptomatic patients, ${ }^{18}$ this proposal has not been incorporated in the established practice guidelines. However, a recent study observed that delay in initiating antibiotic therapy in children with a febrile UTI was associated with the development of renal scarring. ${ }^{19}$ Specifically, the authors reported that a delay of $\geq 48$ hours increased the odds of new renal scarring by approximately $47 \% .^{19}$ This observation may emphasise the importance of rapid pretreatment diagnosis of UTI, or support the commencement of antibiotic therapy in children with febrile UTI without a positive urine culture result.

Identifying the most useful diagnostic test is critical for prompt treatment of UTI. However, there is no common agreement on the most 
reliable single test. Some authors reported that urinalysis had excellent negative predictive value that was not enhanced by urine Gram stain in children with UTI. ${ }^{20}$ Another study observed that point-of-care Gram stain was a useful rapid diagnostic tool for suspected UTI in young children; ${ }^{21}$ it was concluded that pathogentargeted therapy based on the point-of-care Gram stain would result in better antibiotic selection compared with empirical treatment.

In the revised AAP guideline, clinicians are encouraged to first consider the likelihood of a child having a UTI before starting their evaluation, given the fact that some host risk factors increase the likelihood of infection. ${ }^{22}$ The AAP guideline recommendation for the diagnosis of UTI in children aged 2-24 months requires microscopic urinalysis results suggestive of infection (pyuria with or without bacteriuria) and the presence of $\geq 5 \times 10^{4}$ or $50,000 \mathrm{CFU} / \mathrm{mL}$ of a single uropathogen, cultured from a catheter or SPA urine specimen. This investigation can be undertaken once a clinician determines that the pre-evaluation likelihood of UTI merits obtaining a urine culture. The recommendation differs from the previous AAP guideline, which recommended urine testing for all children aged 2-24 months with unexplained febrile illness. ${ }^{23}$ The revised AAP guideline recommends selective urine testing based on the probability of UTI. A set of risk factors help to determine 'low risk' of infection. These factors for females include Caucasian race, age $<12$ months, temperature $\geq 39^{\circ} \mathrm{C}$, fever $\geq 2$ days, and absence of another source of infection. For males, these factors include Caucasian race, temperature $\geq 39^{\circ} \mathrm{C}$, fever $>24$ hours, absence of another source of infection. For both sexes, $\leq 2$ factors are associated with $\leq 1 \%$ risk of $U T I$, and $\leq 3$ is associated with $\leq 2 \%$ risk of UTI. ${ }^{22}$ Caution is advised, however, in the application of this rule because the threshold probability for urine testing is not yet well established. Hence, evaluating each case individually is recommended, rather than urine testing based on certain identified risk factors alone. ${ }^{14}$

The NICE guideline, by comparison, recommends urine microscopy and culture for children aged 3-36 months with specific urinary symptoms, or with nonspecific symptoms who are at high risk of serious illness. ${ }^{15}$ Dipstick urinalysis is recommended in children aged $\geq 3$ years as the initial diagnostic step for UTI. For urine culture results, diagnosis of UTI should be based on a colony count of any Gram-negative bacilli, $>10^{3}$ or 1,000 CFU/mL of a Gram-positive cocci from a SPA specimen, $>10^{5}$ or $10,000 \mathrm{CFU}$ of a single uropathogen per $\mathrm{mL}$ from a catheter urine specimen, or $\geq 10^{5}$ or $10,000 \mathrm{CFU}$ of a single uropathogen per $\mathrm{mL}$ from a clean-catch or midstream urine specimen. ${ }^{15}$

A comparative analysis of the two established guidelines indicates that the revised AAP guideline reduces emphasis from the role of dipstick urinalysis in the diagnosis of UTI. In contrast, the NICE guideline underscores its importance in the initial diagnosis of the infection in older children ( $\geq 3$ years) (Table 1). The NICE guideline provides caveats which relate to the interpretation and implication of dipstick urinalysis results for leucocyte esterase and nitrate testing. ${ }^{15}$ For instance, the sensitivity of nitrite testing is low and has been noted to be $53 \%$, while its specificity is reportedly as high as $98 \%$. Urine should be in the bladder for at least 1 hour for conversion of nitrate to nitrite to occur. In infants, who have short bladder transition time, nitrite testing may be falsely negative, reflecting its low sensitivity in this age group. The NICE guidelines suggest the use of dipstick testing as the initial diagnostic test for infants and children $<3$ years with a suspected UTI, and then if it is positive, the urine should be sent for culture. ${ }^{15}$

Secondly, disparities exist between the two guidelines regarding reference age ranges of pre-toilet-trained children (2-24 months versus 3-36 months), and the bacterial colony counts required for the diagnosis of UTI from the different sources of urine specimen.

The revised AAP guideline recommends the use of microscopic urinalysis findings, such as bacteriuria and pyuria, in conjunction with comparatively lower bacterial colony counts ( $5 \times 10^{4}$ or $50,000 \mathrm{CFU} / \mathrm{mL}$, compared to $>10^{5}$ or $100,000 \mathrm{CFU} / \mathrm{mL}$ recommended by the NICE guideline). Furthermore, the AAP guideline suggests that the absence of pyuria in a true UTI is usually attributable to either a faulty method or insensitive laboratory definition of pyuria. ${ }^{22}$ It maintains that the possibility of pyuria from other febrile illnesses does not preclude the fact that it is rarely absent in a true UTI. 
Table 1: Comparative analysis of recommendations in the revised American Academy of Pediatrics (AAP) ${ }^{14}$ and National Institute for Health and Clinical Excellence (NICE) ${ }^{15}$ guidelines for urinary tract infection in children.

\begin{tabular}{|c|c|c|}
\hline Parameters & AAP guidelines (2011) & NICE guidelines (2007) \\
\hline $\begin{array}{l}\text { Diagnosis of UTI } \\
\text { - Using dipstick urinalysis } \\
\text { - Using microscopic urinalysis and } \\
\text { urine culture }\end{array}$ & $\begin{array}{l} \\
\text { - Bacteriuria with or without pyuria } \\
-\geq 5 \times 10^{4} \text { CFU/mL (from SPA and } \\
\text { catheter urine specimens) }\end{array}$ & $\begin{array}{l}\text { - Positive leucocyte esterase or } \\
\text { nitrite test* } \\
\text { - Colony count of any Gram negative } \\
\text { bacilli or }>10^{3} \mathrm{CFU} / \mathrm{mL} \text { of a Gram- } \\
\text { positive coccus (from SPA urine } \\
\text { specimen) } \\
\text { - }>10^{5} \mathrm{CFU} / \mathrm{mL} \text { (from catheter urine } \\
\text { specimen) } \\
\text { - } \geq 10^{5} \mathrm{CFU} / \mathrm{mL} \text { (from 'clean-catch' or } \\
\text { midstream urine specimen) }\end{array}$ \\
\hline $\begin{array}{l}\text { Radiological investigation of UTI' } \\
\text { - RBUS } \\
\text { - DMSA renal scan } \\
\text { - VCUG (or MCUG) }\end{array}$ & $\begin{array}{l}\text { - Recommended in febrile infants } \\
\text { with first UTI } \\
\text { - Not recommended as routine } \\
\text { investigation for first febrile UTI } \\
\text { - Not recommended as routine } \\
\text { investigation for first febrile UTI }\end{array}$ & $\begin{array}{l}\text { - Recommended in atypical or } \\
\text { recurrent UTI in children aged }<6 \\
\text { months } \\
\text { - Recommended in children aged } 6 \\
\text { months to } 3 \text { years with atypical or } \\
\text { recurrent UTI } \\
\text { - Not recommended in children aged } \\
6 \text { months to } 3 \text { years with atypical or } \\
\text { recurrent UTIf }\end{array}$ \\
\hline $\begin{array}{l}\text { Treatment and follow-up of UTI } \\
\text { - Route of antibiotics/duration }\end{array}$ & $\begin{array}{l}\text { - Parenteral route for } 48 \text { hours (for } \\
\text { critically ill patients) and switch to } \\
\text { oral route if clinical improvement } \\
\text { occurs. } 7-10 \text { days as the total } \\
\text { duration of therapy }\end{array}$ & $\begin{array}{l}\text { - For children aged }<3 \text { months: } \\
\text { parenteral route for } 2-3 \text { days before } \\
\text { a switch to oral route if clinical } \\
\text { improvement occurs. } 10 \text { days as the } \\
\text { total duration of therapy } \\
\text { - For children aged }>3 \text { months } \\
\text { with upper UTI: oral route using } \\
\text { antibiotics with low-resistance } \\
\text { pattern. } 7-14 \text {-day duration of } \\
\text { therapy } \\
\text { - For children aged }>3 \text { months with } \\
\text { lower UTI: oral route for } 3 \text { days }\end{array}$ \\
\hline $\begin{array}{l}\text { - Follow-up routine urine culture } \\
\text { - Follow-up antibiotic prophylaxis }\end{array}$ & $\begin{array}{l}\text { - Not recommended** } \\
\text { - Not recommended }\end{array}$ & $\begin{array}{l}\text { - Not recommended } \\
\text { - Not recommended }\end{array}$ \\
\hline
\end{tabular}

* Initial diagnostic step for UTI in children aged $\geq 3$ years.

+ Top-down approach recommends RBUS and DMSA renal scan as initial radiological investigations of UTI. Bottomup approach recommends MCUG (VCUG) as the initial radiological investigation.

‡ Performed 4-6 months after UTI.

拉 If there is ureteral dilatation on RBUS or poor urine flow or non-Escherichia coli UTI, or family history of VUR.

§ Parenteral route for 2-4 days if the patient is vomiting, then switch to oral route for a total duration of 10 days.

** The previous guideline recommended follow-up urine culture after 48 hours of no clinical improvement.

AAP: American Academy of Pediatrics; CFU: colony-forming units; DMSA: dimercaptosuccinic acid; MCUG: mictuirating cystourethrography; NICE: National Institute for Health and Clinical Excellence; RBUS: renal and bladder ultrasonography; SPA: suprapubic aspiration; UTI: urinary tract infection; VCUG: voiding cystourethrography; VUR: vesico-ureteric reflux. 
In contrast, the NICE guideline suggests that the absence of bacteriuria or pyuria does not completely exclude the diagnosis of UTI. This position on pyuria appears to be evidencebased considering the findings of two studies conducted in children with febrile neutropenia and UTI. ${ }^{24,25}$ In these reports, the sensitivity of pyuria as a diagnostic parameter for UTI was found to be very low. These findings highlight the importance of bacteriuria and urine nitrite testing in this population of children with UTI, as neither of these diagnostic parameters would be affected by the absence of pyuria. ${ }^{26}$ As a result, it seems reasonable to include urine nitrite and leucocyte esterase tests as part of the initial diagnosis of UTI in older children, as recommended by the NICE guideline.

Finally, the recommended techniques for collecting urine samples vary slightly between the two guidelines. The revised AAP guideline emphasises urine sample collection through catheterisation or SPA, while the NICE guideline adds absorbent urine-collection pads to the other options of clean-catch, catheterisation, or SPA samples. In the former, the urine collection technique is restricted to children aged 2-24 months. Recent evidence, however, suggests that the use of catheterisation for infants, and midstream or clean-catch urine (without cleansing the external genitalia for older children), constitute the most reliable methods for obtaining a good urine specimen for culture. ${ }^{26}$ Catheterisation may be contraindicated in certain circumstances: gross infection of the external genitalia, labial adhesions in female children, or failure to visualise the urethral meatus in uncircumcised male children. Similarly, gross contamination of the external genitalia requires proper cleansing in school-aged children before any clean-catch urine collection. ${ }^{27}$

\section{RADIOLOGICAL INVESTIGATION OF CHILDREN WITH URINARY TRACT INFECTION}

Considerations addressed by the established clinical practice guidelines include radiological testing after a diagnosis of UTI, and how affected children should be followed up. Previous recommendations were predicated on evidence that VUR developed from recurrent UTI and subsequently led to renal scarring; therefore, the established guidelines had previously advocated comprehensive radiological investigations to detect possible VUR and renal scarring. ${ }^{23,28}$

The use of radiological investigations for evaluating a child with UTI remain controversial due to their invasive nature and radiation burden, as well as the current movement away from the concept of 'UTI-VUR-renal scar' progression. Two approaches to post-UTI imaging ('topdown' and 'bottom-up' approaches) have arisen. $^{29}$ In the top-down approach, the involvement of renal involvement during UTI helps predict the presence or absence of acute pyelonephritis, renal dysplasia, or acquired renal scarring. ${ }^{5}$ Advocates of the top-down approach recommend initial investigation with renal and bladder ultrasonography (RBUS) and dimercaptosuccinic acid (DMSA) renal scan; VCUG (or MCUG) is performed only if renal involvement is observed. The bottomup approach focusses on bladder involvement during UTI to detect VUR; this makes VCUG (or MCUG) an initial investigation. Although the top-down approach shows a high sensitivity in detecting VUR and renal scarring after a first febrile UTI, it is associated with the highest financial and radiation exposure costs. This drawback casts a doubt on its benefit and highlights the need for the clinician to apply the appropriate protocol that best suits the individual patient under investigation.

Further consideration is needed of the mismatch between VUR grading with VCUG, and ultrasound detection of abnormal morphology. Evidence supports the finding that when sonographic diagnosis of reflux is based solely on morphological criteria and degree of dilatation, correlation with VCUG findings appears poor. Additionally, evidence supports that a positive DMSA scan successfully identifies significant VUR in most instances, which strengthens a possible positive correlation between VCUG reflux grading and DMSA findings. This may support the possibility of using DMSA scan alone as the primary evaluation for children after a UTI episode and reduce use of VCUG in evaluating these children.

The NICE and revised AAP guidelines do not support routine radiological investigations for 
children with first UTI. 15,22 In the NICE guidelines, radiological investigations are recommended depending on different factors: therapeutic response within 48 hours, evidence of atypical UTI, evidence of recurrent UTI, and the age of the child..$^{15}$ The guideline recommends RBUS in cases of atypical UTI, recurrent UTI, or children $<6$ months of age. DMSA renal scan is recommended only in children $<3$ years of age with atypical or recurrent UTI, and it is performed 4-6 months after UTI. By comparison, the revised AAP guideline recommends the performance of RBUS in febrile children aged 2-24 months with first UTI, to detect anatomic or structural abnormalities that may require further evaluation. ${ }^{22}$ The guideline also discourages the use of VCUG (or MCUG) and DMSA as routine investigations after first febrile UTI.

The current practice of a restrictive approach in radiological investigations is supported by evidence from several studies that assessed the clinical importance of renal imaging. ${ }^{30-33}$ The top-down approach in renal imaging, which forms the basis for the recommendations in the NICE and revised AAP guidelines, is supported by the findings of other studies. ${ }^{34-36}$ Although UTI in children usually resolves with no sequelae, the fact that some children are predisposed to recurrence may partly be a reflection of an underlying congenital anomaly of the kidney and urinary tract and urinary tract obstruction, justifying the recommendation for RBUS as the initial imaging study following a first UTI. ${ }^{5}$

The American College of Radiology (ACR) Appropriateness Criteria $^{\circledR}$ rates the following radiological investigations highly in the evaluation of select children with UTI: RBUS, radionuclide cystography, VCUG (or MCUG), and DMSA scan. ${ }^{37}$ RBUS is considered useful for evaluating structural integrity of the kidneys and urinary tract, but is unreliable for detecting VUR. ${ }^{38}$ VCUG (or MCUG) is sufficient for screening and grading VUR, whereas DMSA scan is adequate for evaluating renal scarring. ${ }^{38}$ Thus, radiological investigations that seek to evaluate predisposing factors (structural anomalies) and complicating factors (VUR/ renal scarring) of UTI would seem appropriate, despite the emerging evidence against the UTIVUR-renal scar progression pathway. ${ }^{11-13}$ This $A C R$ rating appears to be the guiding principle in the recommendations of AAP and NICE established guidelines.

\section{TREATMENT AND FOLLOW-UP OF URINARY TRACT INFECTION IN CHILDREN}

The causative bacterial pattern determines the empirical antibiotic treatment of UTI in children. However, with rising antibiotic resistance of common uropathogens, clinicians should stay up-to-date with local bacterial resistance patterns that could influence antibiotic choices. The following factors should guide the choice and route of administration of empirical antibiotics: age of the child, severity of the clinical presentation, location of the infection (upper or lower tract), presence of complications, and prevalence and pattern of local antibiotic resistance. $^{39}$ One study recommended the following antibiotics for empirical therapy in pyelonephritis, according to age group: a combination of aminoglycoside/ampicillin or ceftazidime/ampicillin in early infancy; and an oral third-generation cephalosporin later in infancy and childhood. 39 However, the variable multiregional prevalence rates of extendedspectrum $\beta$-lactamase-producing bacteria have been increasing in acute care settings, and have brought the issue of multiantibiotic resistance to the fore. This trend thus negates the current choice of antibiotics used for empirical therapy.

Since the goals of treating acute UTI are to eliminate the infection, prevent complications, and reduce the likelihood of renal damage, the AAP guideline recommends that clinicians base the choice of antibiotics on local antimicrobial sensitivity patterns (if available) and adjust this choice according to sensitivity testing of the isolated uropathogen. ${ }^{22}$ Moreover, the choice of route of antibiotics should be based on practical considerations as initiating treatment orally or parenterally is equally efficacious. The guideline also advocates for a 7-14-day duration of antimicrobial therapy. A Cochrane review analysed short-duration (2-4 days) versus standard-duration (7-14 days) oral antibiotics in 652 children with lower UTI. ${ }^{40}$ The authors reported no significant difference in positive urine cultures between the two therapies immediately after treatment; ${ }^{40}$ furthermore, there was no significant difference between short and standard duration therapies in the development of resistant organisms after the course of treatment. These findings support 
that a 2-4-day course of oral antibiotics is as effective as a 7-14-day course in children with lower UTI. However, meta-analysis studies suggest that a single-dose or single-day course may be less effective than more prolonged courses of oral antibiotics, and are therefore strongly discouraged. ${ }^{41,42}$

While there is enough evidence to show that most children with UTI can be treated with oral antibiotics, ${ }^{43-45}$ the revised AAP guideline recommends that "patients whom clinicians judge to be 'toxic' or who are unable to retain oral intake (including medications) should receive an antimicrobial agent parenterally until they exhibit clinical improvement (generally within 24-48 hours) and can retain orally administered fluids and medications". ${ }^{22}$ However, oral antibiotics are as effective as parenteral therapy in children. ${ }^{44} \mathrm{~A}$ randomised clinical trial demonstrated no difference in the prevalence of post-UTI renal scarring between children who were treated with oral antibiotics alone and those treated with both parenteral and oral antibiotics. ${ }^{46}$ It is therefore not surprising that current guidelines have generally tilted towards oral antibiotic therapy for UTI in children.

The NICE guideline recommends parenteral antibiotics (precise duration not stated) for UTI in children aged $<3$ months, with 2-3-day duration recommended before switching to oral antibiotics, if there is clinical improvement. ${ }^{15}$ For children aged $>3$ months with upper UTI, oral antibiotics with low resistance patterns are recommended for 7-14 days. If the patient is vomiting, the guideline recommends parenteral antibiotics for 2-4 days and a switch to oral antibiotics for a total duration of 10 days. For children aged $>3$ months with lower UTI, oral antibiotics for 3 days is advised.

Advice for the follow-up of children treated for UTI concerning repeat urine cultures differs between sources. Although several earlier studies support the necessity for routine urine cultures following the commencement of therapy, ${ }^{47-53}$ a more recent study suggests that follow-up urine cultures were not useful in children hospitalised for UTI, including those with fever lasting beyond 48 hours or those with an underlying urological disease. ${ }^{54}$ The previous AAP guideline recommended a repeat urine culture if the expected clinical response (i.e., resolution of fever) was not achieved within the first 48 hours of therapy. ${ }^{21}$ This recommendation implies that fever beyond 48 hours is abnormal and should warrant investigation. However, one report had observed that fever persisted at 48 hours among young children hospitalised for UTI, ${ }^{55}$ suggesting fever beyond 48 hours may not be an appropriate criterion for repeat urine cultures. The current AAP guideline does not advocate routine follow-up urine cultures. The NICE guideline similarly recommends that routine follow-up urine cultures in children who are well are unnecessary. Additionally, follow-up culture is not required in children who do not undergo radiological investigation.

Antibiotic prophylaxis aims to prevent the recurrence of UTI. Recurrent UTI, with or without VUR, is currently the most common reason for long-term antibiotic prophylaxis in infants and children. Other indications include febrile UTI in neonates and infants, and UTI with obstructive lesions. Low-dose nitrofurantoin (1-2 mg/kg once per day), and trimethoprim/ sulfamethoxazole (2 mg/kg/night or $5 \mathrm{mg} /$ $\mathrm{kg}$ twice weekly) are the antibiotics most commonly used in the prevention of UTI in children. Antibiotic prophylaxis can prevent recurrence of UTI, renal scarring, or both in young children following a UTI, with or without VUR. ${ }^{5}$ However, emerging evidence has challenged the practice of follow-up prophylactic antibiotics in affected children. A recent study showed that patients with congenital pelvic-ureteric junction obstruction who were not administered prophylactic antibiotics had developed neither UTI or renal scarring on follow-up. ${ }^{53}$ The efficacy of antibiotic prophylaxis has been judged low in several other studies. ${ }^{57-62}$ The Swedish infant high-grade reflux trial compared the efficacy of continuous antibiotic prophylaxis and endoscopic injection treatment in the management of high-grade VUR in infants. ${ }^{63}$ Similarly, it found that the resolution rate of high-grade VUR with injection treatment was higher than that of continuous antibiotic prophylaxis. This finding further suggests that antibiotic prophylaxis appears less effective in preventing UTI recurrence in children with underlying predisposing factors. Perhaps the current burden of proof against antibiotic prophylaxis has influenced the recommendations of the current clinical 
practice guidelines. The NICE guideline does not recommend routine antibiotic prophylaxis in infants and children after first UTI, ${ }^{15}$ and the recent AAP guideline also discourages the practice after first UTI in children aged 2-24 months. ${ }^{22}$

\section{CONCLUSION}

There is substantial overlap in the recommendations of both AAP and NICE guidelines for the diagnosis, radiological investigation, antibiotic treatment, and followup of UTI in childhood. Subtle differences, however, exist between the two guidelines. An evidence-based paradigm shift on some traditional concepts about UTI in children has influenced the revision and update of these guidelines. Regarding the controversial issue of
'UTI-VUR-renal scar' trajectory, further research is required to establish host and genetic factors that may predispose to renal scarring. In future clinical practice guidelines, these factors should be considered in order to reduce the need for invasive radiological investigations. Finally, a revision of the current recommendations for UTI diagnosis may be necessary. Specifically, it may be reasonable to reduce the diagnostic threshold to $\geq 10^{4}$ or $10,000 \mathrm{CFU} / \mathrm{mL}$ against the revised AAP recommendation of $\geq 5 \times 10^{4}$ or $50,000 \mathrm{CFU} / \mathrm{mL}$, and augment diagnostic testing with a reliable detector of significant pyuria such as the leukocyte esterase test. The leukocyte esterase test would help strengthen the clinical significance of urine culture because of its utility in differentiating true UTI from an inflammatory response to urine contamination or asymptomatic bacteriuria.

\section{References}

1. Shaikh $\mathrm{N}$ et al. Prevalence of urinary tract infection in childhood: a meta-analysis. Pediatr Infect Dis J. 2008;27(4):302-8.

2. Chang SL, Shortliffe LD. Pediatric urinary tract infections. Pediatr Clin North Am. 2006;53(3):379-400.

3. Shaikh $\mathrm{N}$ et al. Identification of children and adolescents at risk of renal scarring after a first urinary tract infection: a meta-analysis with individual patient data. JAMA Pediatr. 2014;168(10):893-900.

4. Lin $\mathrm{KY}$ et al. Acute pyelonephritis and sequelae of renal scar in pediatric first febrile urinary tract infection. Pediatr Nephrol. 2003; 18(4):362-5.

5. Paintsil E. Update on recent guidelines for the management of urinary tract infections in children: the shifting paradigm. Curr Opin Pediatr. 2013;25(1):88-94.

6. Zaffanello M et al. Genetic risk for recurrent urinary tract infections in humans: a systematic review. J Biomed Biotechnol. 2010; 2010:321082.

7. Uwaezuoke $\mathrm{SN}$ et al. The prevalence and risk of urinary tract infection in malnourished children: a systematic review and meta-analysis. BMC Pediatr. 2019; 19:261.

8. Schoen EJ et al. New-born circumcision decreases the incidence and costs of urinary tract infections during the first year of life. Pediatrics. 2000;105(4 Pt 1):789-93.

9. Garin $\mathrm{EH}$ et al. Primary vesicoureteral reflux in childhood. Adv Pediatr. 2002; 49:341-57.

10. Lee $\mathrm{YJ}$ et al. Risk factors for renal scar formation in infants with a first episode of acute pyelonephritis: a prospective clinical study. J Urol. 2012;187(3):1032-6.

11. Montini $\mathrm{G}$ et al. Febrile urinary tract infections in children. N Engl J Med. 2011; 365:239-50.

12. Williams $\mathrm{G}$ et al. Vesicoureteral reflux. J Am Soc Nephrol. 2008;19(5):84762.

13. Ichikawa I et al. Paradigm shift from classic anatomic theories to contemporary cell biological views of CAKUT. Kidney Int. 2002;61(3):88998.

14. Newman TB. The new American Academy of Pediatrics urinary tract infection guideline. Pediatrics. 2011;128(3):572-5.

15. National Institute for Health and Clinical Excellence (NICE). Urinary tract infection in children (CG54). Available at: http://www.nice.org.uk/ CG054. Last accessed: 30 November 2019.

16. Park YS. Renal scar formation after urinary tract infection in children. Korean J Pediatr. 2012;55(10):367-70.

17. Rushton HG. Urinary tract infections in children: epidemiology, evaluation, and management. Pediatr Clin North Am. 1997;44(5):1133-69.

18. Heidrich FJ et al. UTI: diagnosis and evaluation in symptomatic pediatric patients. Clin Pediatr (Phila). 2000;39(8):461-72.

19. Shaikh $\mathrm{N}$ et al. Early antibiotic treatment for pediatric febrile urinary tract infection and renal scarring. JAMA Pediatr. 2016;170(9):848-54.

20. Cantey JB et al. Lack of clinical utility of urine Gram stain for suspected urinary tract infection in pediatric patients. J Clin Microbiol. 2015; 53:1282-5.

21. Yodoshi T et al. Utility of point-ofcare Gram stain by physicians for urinary tract infection in children $\leq 36$ months. Medicine (Baltimore). 2019; 98(14):e15101.

22. Roberts KB. Urinary tract infection: clinical practice guideline for the diagnosis and management of the initial UTI in febrile infants and children 2 to 24 months. Pediatrics. 2011;128(3):595-610.

23. American Academy of Pediatrics, Committee on Quality Improvement, Subcommittee on Urinary Tract Infection. Practice parameter: the diagnosis, treatment, and evaluation of the initial urinary tract infection in febrile infants and young children. Pediatrics. 1999;103(4 Pt 1):843-52.

24. Sandoval $\mathrm{C}$ et al. Urinary tract infections in pediatric oncology patients with fever and neutropenia. Pediatr Hematol Oncol. 2012;29(1):6872.

25. Klaassen IL et al. 2011. Pyuria is absent during urinary tract infections in neutropenic patients. Pediatr Blood Cancer. 2011; 56:868-70. 
26. Doern CD, Richardson SE. Diagnosis of urinary tract infections in children. J Clin Microbiol. 2016; 54:2233-42.

27. Lohr JA et al. Hospital-acquired urinary tract infection. Pediatrics. 1989; 83:193-9.

28. Smellie JM et al. Medical versus surgical treatment in children with severe bilateral vesicoureteric reflux and bilateral nephropathy: a randomised trial. Lancet. 2001;357(9265):1329-33.

29. Routh JC et al. Vesicoureteral reflux: current trends in diagnosis, screening, and treatment. Eur Urol. 2012;61(4):773-82.

30. Schroeder AR et al. Impact of a more restrictive approach to urinary tract imaging after febrile urinary tract infection. Arch Pediatr Adolesc Med. 2011; 165:1027-32.

31. Hoberman A et al. Imaging studies after a first febrile urinary tract infection in young children. N Engl J Med. 2003;348(3):195-202.

32. Jahnukainen $\mathrm{T}$ et al. Ultrasonography after the first febrile urinary tract infection in children. Eur J Pediatr. 2006;165(8):556-9.

33. Pennesi $M$ et al. Managing children under 36 months of age with febrile urinary tract infection: a new approach. Pediatr Nephrol. 2012; 27:611-5.

34. Lee JH et al. Is a routine voiding cystourethrogram necessary in children after the first febrile urinary tract infection? Acta Paediatr. 2012; 101:e105-9.

35. Hannula A et al. Imaging the urinary tract in children with urinary tract infection. Acta Paediatr. 2011; 100(12):e253-9.

36. Tsai JD et al. Screening high-grade vesicoureteral reflux in young infants with a febrile urinary tract infection. Pediatr Nephrol. 2012; 27:955-63.

37. American College of Radiology. ACR Appropriateness Criteria: urinary tract infection-child. Available at: https://acsearch.acr.org/docs/69444/ Narrative/. Last accessed: 30 November 2019.

38. White B. Diagnosis and treatment of urinary tract infections in children.
Am Fam Physician. 2011;83(4):409-15.

39. Beetz R, Westenfelder $M$ Antimicrobial therapy of urinary tract infections in children. Int $\mathrm{J}$ Antimicrob Agents. 2011; 38:42-50.

40. Michael M et al. Short versus standard duration oral antibiotic therapy for acute urinary tract infection in children. Cochrane Database Syst Rev. 2003:(1):CD003966.

41. Tran D et al. Short-course versus conventional length antimicrobial therapy for uncomplicated lower urinary tract infections in children a meta-analysis of 1279 patients. J Pediatr. 2001;139(1):93-9.

42. Keren R, Chan E. A meta-analysis of randomized, controlled trials comparing short- and long-course antibiotic therapy for urinary tract infections in children. Pediatrics. 2002; 109(5):E70.

43. Hoberman A et al. Oral versus initial intravenous therapy for urinary tract infections in young febrile children. Pediatrics. 1999;104(1 Pt 1):79-86.

44. Hodson EM et al. Antibiotics for acute pyelonephritis in children. Cochrane Database Syst Rev. 2007;(4):CD003772.

45. Bloomfield P et al. Antibiotics for acute pyelonephritis in children. Cochrane Database Syst Rev. 2005;(1):CD003772

46. Bocquet $\mathrm{N}$ et al. Randomized trial of oral versus sequential IV/oral antibiotic for acute pyelonephritis in children. Pediatrics. 2012; 129:e26975.

47. Ansari BM et al. Urinary tract infection in children, part 1: epidemiology, natural history, diagnosis, and management. J Infect. 1995;30(1):3-6

48. Winberg $\mathrm{J}$ et al. Epidemiology of symptomatic urinary tract infection in childhood. Acta Paediatr Scand Suppl. 1974; 252:1-20.

49. Johnson CE. New advances in childhood urinary tract infections. Pediatr Rev. 1999;20(10):335-43.

50. Shapiro ED. Infections of the urinary tract. Pediatr Infect Dis J. 1992;11(2):165-8.
51. Watson AR. Urinary tract infection in early childhood. J Antimicrob Chemother. 1994;34(Suppl A):53-60.

52. Feld LG et al. Urinary tract infections in infants and children. Pediatr Rev. 1989; 11:71-7.

53. Lerner GR. Urinary tract infections in children. Pediatr Ann. 1994;23(9):46673.

54. Currie $M L$ et al. Follow-up urine cultures and fever in children with urinary tract infection. Arch Pediatr Adolesc Med. 2003; 157:1237-40.

55. Bachur R. Nonresponders: prolonged fever among infants with urinary tract infections. Pediatrics. 2000; 105(5):e59.

56. Islek $A$ et al. Probability of urinary tract infection in infants with ureteropelvic junction obstruction: is antibacterial prophylaxis really needed? Pediatr Nephrol. 2011; 26:1837-41.

57. Montini G et al. Prophylaxis after first febrile urinary tract infection in children? A multicenter, randomized, controlled, non-inferiority trial. Pediatrics. 2008;122(5):1064-71.

58. Song SH, Kim KS. Antibiotic prophylaxis in pediatric urology. Indian J Urol. 2008;24(2):145-9.

59. Le Saux $\mathrm{N}$ et al. Evaluating the benefits of antimicrobial prophylaxis to prevent urinary tract infections in children: a systematic review. CMAJ. 2000;163(5):523-9.

60. Faust WC, Pohl HG. Role of prophylaxis in vesicoureteral reflux. Curr Opin Urol. 2007;17(4):252-6.

61. Hewitt IK et al. Antibiotic prophylaxis for urinary tract infection-related renal scarring: a systematic review. Pediatrics. 2017; 139(5):e20163145.

62. Pennesi $M$ et al. Is antibiotic prophylaxis in children with vesicoureteral reflux effective in preventing pyelonephritis and renal scars? A randomized, controlled trial. Pediatrics. 2008; 121(6):e1489-94.

63. Nordenström J et al. The Swedish infant high-grade reflux trial: Study presentation and vesicoureteral reflux outcome. J Pediatr Urol. 2017;13(2):130-8. 